\title{
Activation of STAT3 signaling pathway in the kidney of COVID-19 patients
}

\author{
Fadi Salem ${ }^{1} \cdot$ Xue Zhu Li ${ }^{2}$. Judy Hindi ${ }^{3}$. Nitzy Munoz Casablanca ${ }^{3} \cdot$ Fang Zhong $^{3}$ - Siraj M. El Jamal ${ }^{1}$. \\ Mohamed Rizwan Haroon Al Rasheed ${ }^{1} \cdot \mathrm{Li} \mathrm{Li}^{1,6} \cdot \mathrm{Kyung} \mathrm{Lee}^{3} \cdot$ Lili Chan $^{3,4} \cdot$ John Cijiang He $\mathrm{H}^{3,5}$
}

Received: 19 April 2021 / Accepted: 17 September 2021 / Published online: 9 October 2021

(c) Italian Society of Nephrology 2021

\begin{abstract}
Background Acute kidney injury is common in patients with COVID-19, however mechanisms of kidney injury remain unclear. Since cytokine storm is likely a cause of AKI and glomerular disease, we investigated the two major transcription factors, STAT3 and NF-kB, which are known to be activated by cytokines.

Methods This is an observational study of the postmortem kidneys of 50 patients who died with COVID-19 in the Mount Sinai Hospital during the first pandemic surge. All samples were reviewed under light microscopy, electron microscopy, and immunofluorescence by trained renal pathologists. In situ hybridization evaluation for SARS-CoV-2 and immunostaining of transcription factors STAT3 and NF-kB were performed.

Results Consistent with previous findings, acute tubular injury was the major pathological finding, together with global or focal glomerulosclerosis. We were not able to detect SARS-CoV-2 in kidney cells. ACE2 expression was reduced in the tubular cells of patients who died with COVID-19 and did not co-localize with TMPRSS2. SARS-CoV-2 was identified occasionally in the mononuclear cells in the peritubular capillary and interstitium. STAT3 phosphorylation at Tyr705 was increased in 2 cases in the glomeruli and in 3 cases in the tubulointerstitial compartments. Interestingly, STAT3 phosphorylation at Ser727 increased in 9 cases but only in the tubulointerstitial compartment. A significant increase in NF-kB phosphorylation at Ser276 was also found in the tubulointerstitium of the two patients with increased p-STAT3 (Tyr705).

Conclusions Our findings suggest that, instead of tyrosine phosphorylation, serine phosphorylation of STAT3 is commonly activated in the kidney of patients with COVID-19.
\end{abstract}

Keywords COVID-19 $\cdot$ Renal pathology $\cdot$ Cytokines $\cdot$ Autopsy

Fadi Salem, Xue Zhu Li and Judy Hindi contributed equally to the study.

\section{Lili Chan \\ lili.chan@mountsinai.org \\ $\triangle$ John Cijiang He \\ Cijiang.he@mssm.edu}

1 Department of Pathology, Molecular and Cell Based Medicine, Icahn School of Medicine at Mount Sinai, New York, NY, USA

2 Department of Nephrology, Shanghai Ninth People's Hospital, Shanghai Jiao Tong University, School of Medicine, Shanghai, China

\section{Introduction}

The Coronavirus Disease 2019 (COVID-19) caused by severe acute respiratory syndrome coronavirus 2 (SARS-CoV-2) has had a devastating impact worldwide. Acute kidney injury (AKI) is a common complication of

3 Division of Nephrology, Department of Medicine, Icahn School of Medicine at Mount Sinai, New York, NY, USA

4 Charles Bronfman Institute of Personalized Medicine, Icahn School of Medicine at Mount Sinai, New York, NY, USA

5 Renal Program, James J Peters VAMC, Bronx, NY, USA

6 Department of Pathology, Anatomy and Cell Biology, Thomas Jefferson University Hospital, Philadelphia, PA, USA 
hospitalized patients with COVID-19 [1]. Urinary abnormalities, such as hematuria, proteinuria, and leukocyturia, are common and affect $90 \%$ of patients regardless of whether they have AKI or not. Acute tubular injury (ATI) has been confirmed in several autopsy reports [2, 3]. Kidney biopsies of COVID-19 patients with significant proteinuria revealed de novo glomerulopathies such as collapsing focal segmental glomerulosclerosis (FSGS), minimal change disease, and membranous nephropathy [4, 5]. However, the mechanisms of kidney injury in COVID19 patients remain unclear.

Given the systemic response to viral infection, the causes of AKI are likely multifactorial, such as cytokine storm, organ-organ crosstalk, and drug toxicity, although direct viral infection of kidney cells likely also contributes to AKI [6]. Initial reports suggested local viral infection of kidney cells such as proximal tubular epithelial cells and podocytes $[2,3]$. However, these findings have been questioned by several renal pathologists [7] and a series of renal biopsy reports did not reveal virus in the kidney cells $[4,5]$. These discrepancies could result from sampling issues due to the focal nature of the infection, and the timing of sampling due to the nature of the transient viral infection. Indeed, a recent report established the presence of virus in kidney cells, and that viral copy number in kidney cells is associated with AKI and overall outcomes [8,9]. Nevertheless, the role of direct viral infection of kidney cells in the development of AKI in COVID-19 patients remains unclear. It is possible that systemic factors, such as circulating cytokines and local infection, contribute to the pathogenesis of AKI in these patients in a synergistic manner.

Here, we report a kidney autopsy series of cases infected with SARS-CoV-2 at the Mount Sinai Hospital. We describe the clinical data and kidney histologic findings in these patients. In addition, we explored potential downstream signaling pathways activated in the kidney of these patients.

\section{Methods}

\section{Autopsy and tissue collection}

Autopsies were performed at the Mount Sinai Hospital in a negative pressure room, utilizing appropriate personal protective equipment (PPE) and techniques as recommended by the Centers for Disease Control and Prevention (CDC). Pre-mortem clinician-collected nasopharyngeal swab specimens were placed in universal transport media and tested by real-time reverse transcription polymerase chain reaction (RT-PCR) amplification using the Cobas 6800 SARS-CoV-2 (Roche Molecular Systems, Branchburg, NJ). Entire kidneys were procured as part of a full autopsy.

\section{Clinical data}

We included any kidney pathology results from autopsies of patients who died with COVID-19 between 3/20/2020 and $6 / 23 / 2020$. Consent for autopsy was obtained from the patients' healthcare proxies. Clinical and laboratory data were manually extracted from the electronic health record (EHR). Clinical data included demographics and comorbidities as identified from the problem list. Chronic kidney disease (CKD) was defined as an estimated glomerular filtration rate $<60 \mathrm{~mL} / \mathrm{min} / 1.73 \mathrm{~m}^{2}$ on two separate occasions or a documented history of CKD. AKI was defined as per KDIGO creatinine criteria. Patients were excluded if they had kidney failure on maintenance dialysis or had a kidney transplant. This study was approved by the Mount Sinai IRB (IRB-20-00523).

\section{Histology and additional studies}

One tissue section was collected from each kidney and stained with hematoxylin and eosin in addition to periodic acid-Schiff, Masson trichrome, and Jones methenamine silver stain. Kidney tissue sections were evaluated by renal pathologists. Autolysis occurs when there is a long postmortem interval or during elevated temperatures. Cases with severe autolytic changes observed in $75 \%$ of kidney parenchyma precluded an accurate assessment of tubular injury, hence were excluded from this study.

\section{RNA in-situ hybridization (IN-SITU)}

RNA IN-SITU was manually performed using the RNAscope ${ }^{\circledR}$ HPV kit (Advanced Cell Diagnostics, Inc., Hayward, CA) according to the manufacturer's instructions. Briefly, $4 \mu \mathrm{m}$ formalin-fixed and paraffin-embedded tissue sections were pretreated with heat and protease prior to hybridization. Tissue sections were hybridized separately with both sense (catalogue\# 848561) and anti-sense (catalogue\# 845701) probes to the SARS-CoV-2 S gene encoding the spike protein. The preamplifier, amplifier, and horseradin-situ peroxidase (HRP)-labeled probes were then hybridized sequentially, followed by color development with DAB. Specific staining signals were identified as brown, punctate dots present in the cytoplasm and/or nucleus. A general RNA probe was used to assess the overall RNA integrity in the viral infected cell line.

\section{Immunofluorescence}

Immunofluorescence studies were performed on $4 \mu \mathrm{m}$ thick formalin-fixed paraffin-embedded tissue slides following 
antigen retrieval in boiling citrate buffer $\mathrm{pH} 6$ (Vector labs), blocking for $1 \mathrm{~h}$ at room temperature [10\% normal donkey serum (NDS) $/ 0.5 \%$ triton-X (TX)], primary antibody incubation overnight at $4{ }^{\circ} \mathrm{C}$ with anti-SARS-Cov-2 nucleocapsid antibody (ProSci, 35-579, 1:100) and species-appropriate Cy3-conjugated secondary antibody incubation for $4 \mathrm{~h}$ at room temperature ( $1 \%$ NDS $/ 0.25 \%$ TX). Nuclear counterstain was with 4',6-diamidino-2-phenylindole (DAPI). Images were visualized on a Zeiss LSM710 confocal microscope.

\section{Transmission electron microscopy (TEM)}

Tissue samples for electron microscopy were first received in neutral buffered formalin, then transferred to $3 \%$ buffered glutaraldehyde. Following post-fixation in $1 \%$ osmium tetroxide, tissues were serially dehydrated and embedded in epoxy resin in standard fashion. One micron of toluidinestained scout sections was prepared for light microscopic orientation and $80 \mathrm{~nm}$ ultrathin sections for EM were stained with uranyl acetate and lead citrate and examined in a Hitachi 7650 transmission electron microscope at $80 \mathrm{kV}$.

\section{Immunohistochemistry (IHC)}

Renal tissues were fixed in $10 \%$ formalin, dehydrated in ethanol, and embedded in paraffin. $3 \mu \mathrm{m}$ thick sections were used for IHC. Paraffin-embedded sections were incubated with primary antibodies and then with biotinylated secondary antibodies, followed by incubation with an avidin-biotin-peroxidase complex and were developed using DAB substrate. (Biotinylated secondary antibody, avidin-biotin-peroxidase complex and DAB substrate were provided by Vector Laboratories Inc.). The following antibodies were used in this study: phospho-STAT3 (Tyr705) (ab76315, Abcam, 1:200), phospho-STAT3 (Ser727) (ab30647, Abcam, 1:200), phospho-NF-kB p65 (Ser276) (ab194726, Abcam, 1:200), NF-kB p65 (ab16502, Abcam, 1:500), ACE2 (ab15348, Abcam, 1:500), and TMPRSS2 (ab92323, Abcam, 1:500). Eleven cases of COVID-19-associated AKI were immunostained for p-STAT3 (Tyr705), p-STAT3 (Ser727), p-NF-KB p65 (Ser276), NF-kB p65, ACE2, and TMPRSS2. Three cases of normal kidney tissue from the uninvolved pole of carcinoma nephrectomy samples, five cases of kidney tissue from non-COVID-19 autopsy cases, and five cases from pre-transplant donor biopsies were used as control.

\section{Quantification of p-STAT3 staining}

The number of p-STAT3 (Tyr705) and p-STAT3 (Ser727) positive cells in glomeruli were counted in at least 20 glomeruli that did not exhibit sclerosis, and data are presented as the mean cell number \pm SD per glomerular cross-section. The number of p-STAT3 (Tyr705) and p-STAT3 (Ser727) positive cells in the tubules and the interstitium were counted in 20 high-power $(\times 400)$ fields (avoiding glomeruli and large vessels) and data are presented as the mean cell number \pm SD per $\mathrm{mm}^{2}$. All counting was performed by observers who were blinded to this study.

\section{Results}

\section{Clinical findings}

Of 94 autopsy cases, 50 met our inclusion and exclusion criteria (Supplemental Figure S1). The mean age was $67 \pm 14$ years old. Twenty-five percent were black and $20 \%$ were white. Twenty-eight percent had diabetes, $58 \%$ had hypertension, $22 \%$ had CKD and $12 \%$ had coronary artery disease (Table 1). AKI occurred in $70 \%$ of patients. The mean days from hospital admission to death was $21 \pm 23$ days.

\section{Histological findings under light microscopy}

The most common finding in the glomeruli was arterionephrosclerosis with a range of globally sclerosed glomeruli from 10 to $\geq 90 \%$ in advanced cases (Supplemental Table S1). Three cases showed segmentally sclerosed glomeruli (Fig. 1A) secondary to ischemia or associated with diabetic changes. Ischemic changes such as ischemic collapse of capillary tufts and wrinkling capillaries were readily seen; however, the collapsing variant of FSGS was not observed. Thirteen cases showed glomerular mesangial expansion and six cases had mesangial nodular sclerosis (Fig. 1B), reflecting the prevalence of hypertension and diabetes among this group. Changes suggestive of immune complex deposition in the glomeruli were not seen by light microscopy. There was frequent congestion of glomerular and peritubular capillaries with focal aggregation of platelets without evidence of thrombotic microangiopathy.

The most common finding of the tubules was ATI characterized by attenuation of tubular epithelial cells and loss of brush borders, which was seen diffusely in sixteen cases (Fig. 1C). Furthermore, diffuse clear vacuolization (isometric) of epithelial cells of proximal tubules was observed in seven cases (Fig. 1D). There was no evidence of viral cytopathic effects found in the tubules. Calcium phosphate crystals were seen in the distal tubules of 10 patients, of which three were diffuse (Fig. 1E). A few cases showed brown discoloration including wear and tear of epithelial cells in addition to tubular casts.

Arteriosclerosis was seen in $43 / 50$ cases and showed a spectrum of mild to severe intimal fibrosis which correlated 
Table 1 Patient characteristics

\begin{tabular}{|c|c|}
\hline Patient characteristic & $\begin{array}{l}\text { Patients who } \\
\text { underwent autopsy } \\
(\mathrm{N}=50)\end{array}$ \\
\hline Age, years $($ mean $\pm S D)$ & $67 \pm 14$ \\
\hline Female & $24(48)$ \\
\hline \multicolumn{2}{|l|}{ Race (\%) } \\
\hline White & $10(20)$ \\
\hline Black & $12(25)$ \\
\hline Asian & $2(4)$ \\
\hline Other/unknown & $25(51)$ \\
\hline \multicolumn{2}{|l|}{ Comorbidities (\%) } \\
\hline Hypertension & $29(58)$ \\
\hline Diabetes mellitus & $14(28)$ \\
\hline Chronic kidney disease & $11(22)$ \\
\hline Coronary artery disease & $6(12)$ \\
\hline Chronic obstructive pulmonary disease & $1(2)$ \\
\hline Asthma & $7(14)$ \\
\hline \multicolumn{2}{|l|}{ Hospital events (\%) } \\
\hline AKI & $35(75)$ \\
\hline ICU admission & $26(47)$ \\
\hline Dialysis & $7(47)$ \\
\hline Mechanical ventilation & $28(60)$ \\
\hline \multicolumn{2}{|l|}{ Laboratory Results (mean $\pm \mathrm{SD}$ ) } \\
\hline $\mathrm{WBC}\left(10^{3} / \mu \mathrm{L}\right)$ & $12.1 \pm 6.4$ \\
\hline Hemoglobin (g/dL) & $11.0 \pm 2.5$ \\
\hline Lymphocyte Percent (\%) & $10 \pm 7$ \\
\hline Creatinine $(\mathrm{mg} / \mathrm{dL})$ & $2.2 \pm 1.6$ \\
\hline Phosphorus (mg/dL) & $4.2 \pm 1.2$ \\
\hline Creatine phosphokinase (units/L) & $354 \pm 386$ \\
\hline Uric Acid (mg/dL) & $8.6 \pm 3$ \\
\hline D-dimer (ng/mL) & $5.5 \pm 5.3$ \\
\hline Fibrinogen (mg/dL) & $631 \pm 202$ \\
\hline Ferritin $(\mathrm{ng} / \mathrm{mL})$ & $1670 \pm 2626$ \\
\hline Interleukin 6 (pg/mL) & $797 \pm 3155$ \\
\hline
\end{tabular}

to the severity of hypertension and the age of this cohort. Arteriolar hyalinosis was a constant finding in cases with diabetes. Two of five patients with postmortem findings of a pulmonary embolism showed thromboemboli in small arcuate arteries (Fig. 1F).

\section{Transmission electron microscopy findings}

Cases with diabetes showed variable mesangial scleroses and thickening of glomerular basement membranes in proportion to the severity of diabetic nephropathy. One case showed subepithelial immune type electron dense deposits in the glomeruli. No deposits are seen in the tubular basement membranes or interstitium (Fig. 1G).
Viral or "viral-like particles" were not present in proximal tubules or glomerular endothelial cells [10, 11]. Tubuloreticular inclusions were not observed [12].

\section{Immunohistochemistry, immunofluorescence and In-situ hybridization (RNAscope) for SARS-CoV-2}

Immunohistochemistry and immunofluorescence studies showed expression of SARS-CoV-2 in mononuclear white blood cells within the peritubular capillaries and the interstitium. However, no evidence of SARS-CoV-2 was seen in tubular epithelial cells or glomeruli (Fig. 1H).

In-situ hybridization (RNAscope) of several cases was negative, however, one case showed scattered rare staining which was indefinite for direct viral infection (Fig. 1I).

\section{ACE2 and TMPRSS2 expression}

ACE2, the receptor of SARS-CoV-2, was expressed strongly in the apical aspect of proximal tubules and parietal epithelial cells, but podocytes showed only occasional weak positive staining. TMPRSS2, the cleavage enzyme of the viral Spike protein, was robustly expressed in the distal tubules, but not in the proximal tubules (Supplemental Figure S2). Additionally, staining of consecutive kidney sections did not find co-localization of ACE2 or TMPRSS2 in the glomeruli or tubules; which is consistent with previous single-cell transcriptomic data [13] and with the low viral infection rate in the kidney. Compared with normal kidneys, the expression of ACE2 in the tubules of cases of COVID-19-associated AKI was decreased but there was not a significant increase in TMPRSS2 (Supplemental Figure S3). These data suggest that ACE2 protein levels are downregulated in COVID-19 patients, similar to previous findings in the lung [14].

\section{STAT3 activation in COVID-19-associated AKI}

Since it is known that IL-6 is highly upregulated in patients with COVID-19, we sought to determine whether its downstream signaling molecule, STAT3, was activated in the kidney of patients with COVID-19. The JAK-STAT3 pathway is well-known to be involved in the pathogenesis of both acute and chronic kidney injury [15]. We selected 11 cases for further study of signaling molecules activated in the COVID-19 kidney (Supplemental Table S2).

Control human kidney tissue from non-neoplastic renal parenchyma and non-COVID-19 autopsy kidney samples exhibited small basal numbers of STAT3 phosphorylation (p-STAT3) at either tyrosine (Tyr705) or serine (Ser727) sites in both glomerular and tubulointerstitial cells (Fig. 2 and Supplemental Figure S4). Compared with normal control kidney and non-COVID-19 autopsy kidney, two cases with COVID-19-associated AKI showed a significant 

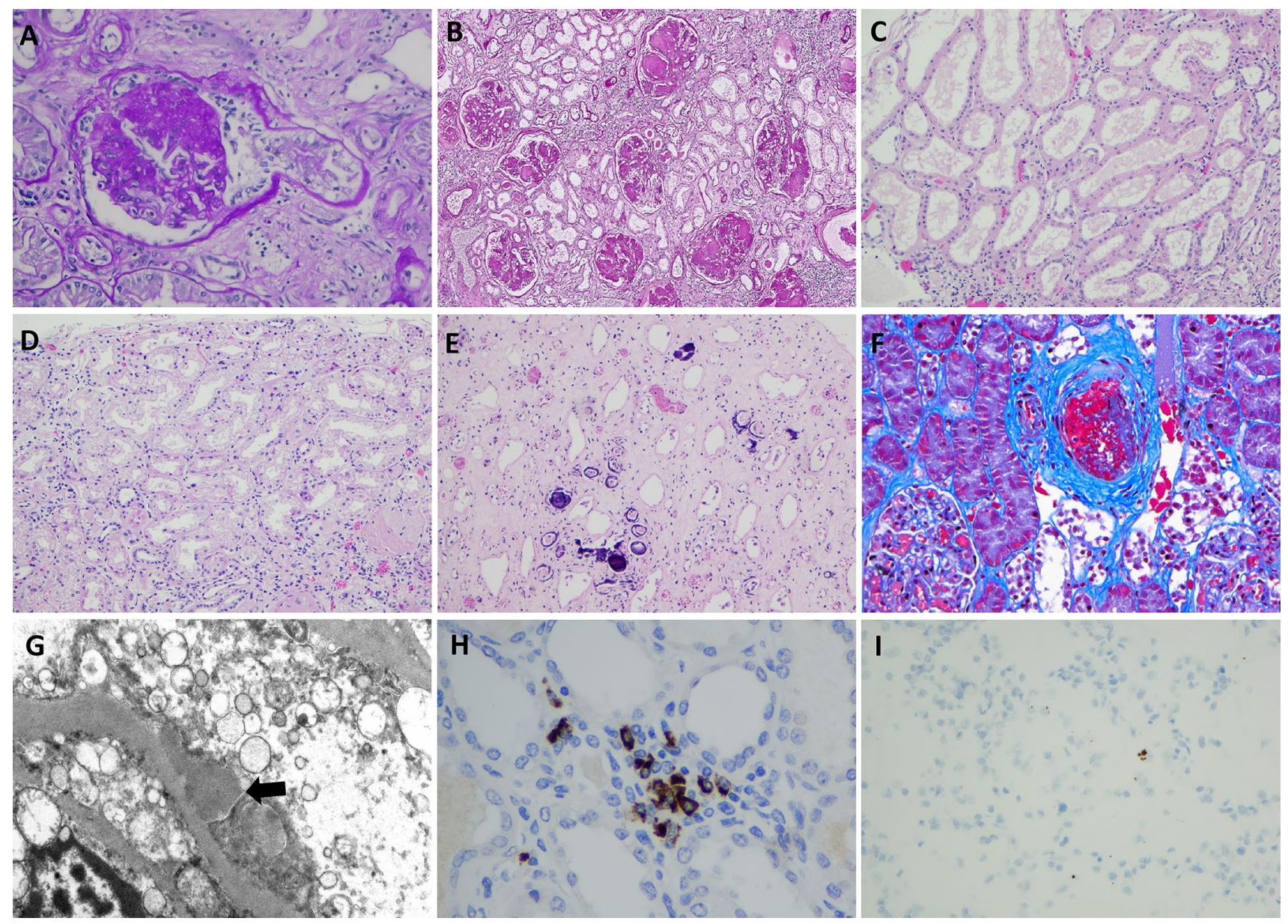

Fig. 1 Histologic findings in kidney from postmortem patients with COVID-19. A Segmental obliteration of capillary tufts and prominence of epithelial cells consistent with focal segmental glomerulosclerosis $($ FSGS) $(\mathrm{PAS} \times 200)$. B Diffuse mesangial nodular sclerosis in a case of diabetic nephropathy (PAS $\times 100)$. C Proximal tubules show diffuse attenuation of epithelial cells consistent with acute tubular injury $(H \& E \times 100)$. D Acute tubular injury associated with vacuolization of proximal tubules $(\mathrm{H} \& \mathrm{E} \times 200)$. E Calcium phosphate crystals identified within tubules $(\mathrm{H} \& \mathrm{E} \times 100)$. F An arcuate artery shows bright red intraluminal thrombus (Gomori Trichrome $\times 200$ ). G Electron microscopy shows subepithelial immune-type electron dense deposits (Transmission electron microscopy $\times 7000$ ). H IHC stain for SARS-COV-2 spike protein showing granular staining of mononuclear cells in peritubular capillaries $(\times 200)$. I Rare positive staining of SARS-CoV-2 by RNAscope $(\times 100)$ increase in the number of p-STAT3 (Tyr705)-positive cells in glomeruli and three cases showed a significant increase in tubulointerstitial cells (Fig. 2 and Supplemental Figure S4 a, b). While there was no increase in p-STAT3 (Ser727) positive glomerular cells in COVID-19-associated AKI, 9 of 11 cases exhibited a significant increase in the number of p-STAT3 (Ser727)-positive cells in the tubulointerstitium (Fig. 2 and Supplemental Figure S4c, d), and p-STAT3 (Ser727) activation was seen in all tubular segments. These data suggest that tyrosine phosphorylation of STAT3 occurs in both glomerular and tubular compartments of only a few cases while serine phosphorylation of STAT3 is observed in most cases, but only in the tubulointerstitial compartments.

\section{STAT3 activation in COVID-19 lung tissue}

To confirm whether a similar pattern of STAT3 phosphorylation occurs in the lung of COVID-19 patients, we evaluated the expression of p-STAT3 (Tyr705) and p-STAT3 (Ser727) in 2 cases of COVID-19 positive lung tissue. There was a significant increase of p-STAT3 (Tyr705)-positive pneumocytes and inflammatory cells in the lung. In particular, numerous macrophages showed robustly positive staining for p-STAT3 (Tyr705). However, the staining pattern of p-STAT3 (Ser727) was localized mostly in pneumocytes and endothelial cells of small vessels (Supplemental Figure S5). 
Healthy Kidney Donor Biopsy Non-COVID-19 Autopsy
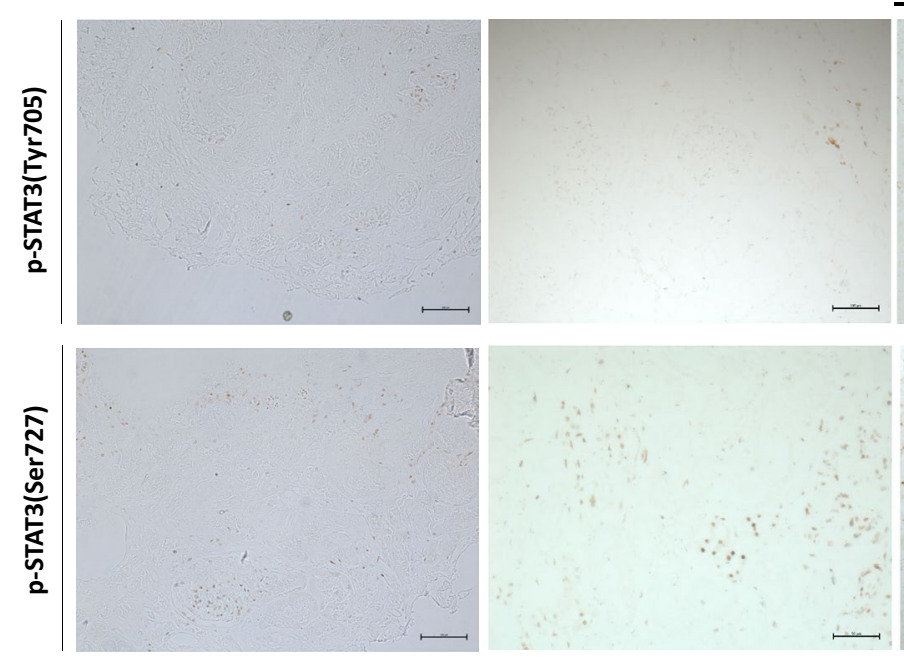

Fig. 2 Glomerular and tubulointerstitial phosphorylated signal transducer and activator of transcription 3 (p-STAT3) expression in normal and COVID-19-associated AKI kidney. Normal and nonCOVID-19 autopsy kidney shows a small amount of p-STAT3 (both Tyr705 and Ser727) positive staining in glomeruli, tubules and inter-

\section{NF-KB activation and translocation}

TNF- $\alpha$ is also highly upregulated in COVID-19 patients and TNF- $\alpha / \mathrm{NF}-\mathrm{kB}$ also plays a major role in the pathogenesis of kidney disease [16]. NF-kB has been considered as a therapeutic target for COVID-19 patients [17]. Therefore, we evaluated whether the NF-kB pathway was activated in the kidney of COVID-19 patients. p-NF- $\kappa B$ (Ser276) and total NF- $\mathrm{kB}$ immunostaining were negative in the normal control kidney. However, there was a significant increase in the number of p-NF-kB (Ser276)-positive cells in the tubulointerstitium in the two COVID-19 cases which also had increased p-STAT3 (Tyr705) staining. Consistent with this, the total NF- $\mathrm{KB}$ staining also showed the translocation from cytoplasm to nuclei (Fig. 3). These data suggest that NF-kB and tyrosine phosphorylation of STAT3 are activated in the same patients, indicating they might be activating each other through a positive feedback mechanism.

\section{Discussion}

In one of the largest series of kidney autopsy cases, we found ATI was the predominant pathology and that these patients also had significant glomerular injuries related to comorbidities or ischemic changes, which is consistent with other autopsy series [3, 18, 19]. Thrombotic injury was not common in the kidney. STAT3 and NF- kB pathways were both activated in the kidney of COVID-19 samples. While not found here, prior autopsy and kidney biopsy series in
COVID-19 Autopsy

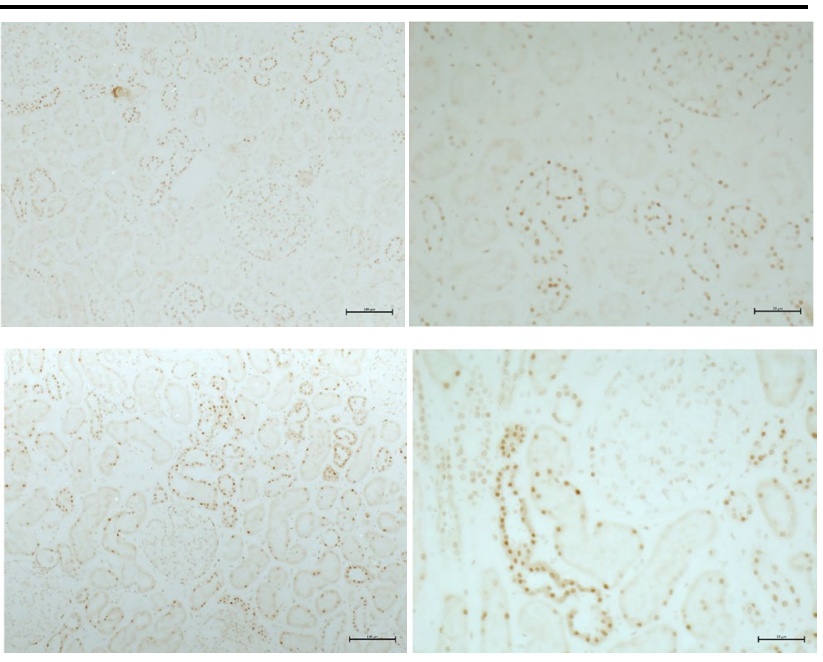

stitium. Compared with normal kidney and non-COVID-19 autopsy kidney, there was an increase in glomerular and tubulointerstitial p-STAT3 (Tyr705) and p-STAT3 (Ser727) staining in kidney from cases with COVID-19-associated AKI kidney

COVID-19 patients with AKI who had severe proteinuria identified multiple glomerular diseases such as collapsing FSGS in patients with APOL1 risk alleles, minimal change disease, and lupus nephritis [4, 20-22]. Therefore, there is a broad spectrum of COVID-19-associated kidney disease.

The mechanism of kidney injury in COVID-19 patients appears complicated and remains unclear. Our current findings suggest a potential mechanism of kidney cell injury induced by SARS-CoV-2 through activation of STAT3 and NF-kB. Cytokine storm is a characteristic feature of severe COVID-19 disease with significant elevations of multiple cytokines including IL-6, TNF- $\alpha$, and interferons $(\alpha, \beta, \gamma)$. It is known that these cytokines play an important role in the pathogenesis of both AKI and glomerular injury [15, 16, 23-26]. Additionally, activation of STAT3 and NF-kB has been implicated in sepsis and ischemia-reperfusionassociated AKI models [27-29]. NF-kB and STAT3 are key downstream transcription factors induced by these cytokines and known to be involved in the pathogenesis of kidney disease [16, 30-33].

Whether local infection of kidney cells by SARS-CoV-2 contributes to the development of AKI and glomerular disease remains controversial. Like others, we did not identify characteristic findings of viral infection in the kidney [4, $5,19]$. While we demonstrated infection of SARS-CoV-2 in the mononuclear white blood cells within peritubular capillaries and the interstitium, it remains unclear whether these positive cells could explain the positive PCR findings in the COVID-19 kidney [4], or if these infiltrated cells may infect kidney cells indirectly. Current findings suggest that 
Fig. 3 Immunostaining of phosphorylated nuclear factor kappa-light-chain-enhancer of activated $\mathrm{B}$ cells ( $\mathrm{p}-\mathrm{NF}-\mathrm{\kappa B})$ (Ser276) expression in kidney donor and COVID-19-associated AKI kidney. The expression of $\mathrm{p}-\mathrm{NF}-\mathrm{\kappa B}(\operatorname{Ser} 276)$ also significantly increased, and total $\mathrm{NF}-\kappa \mathrm{B}$ showed translocation from cytoplasm to nuclei in STAT3 activation cases
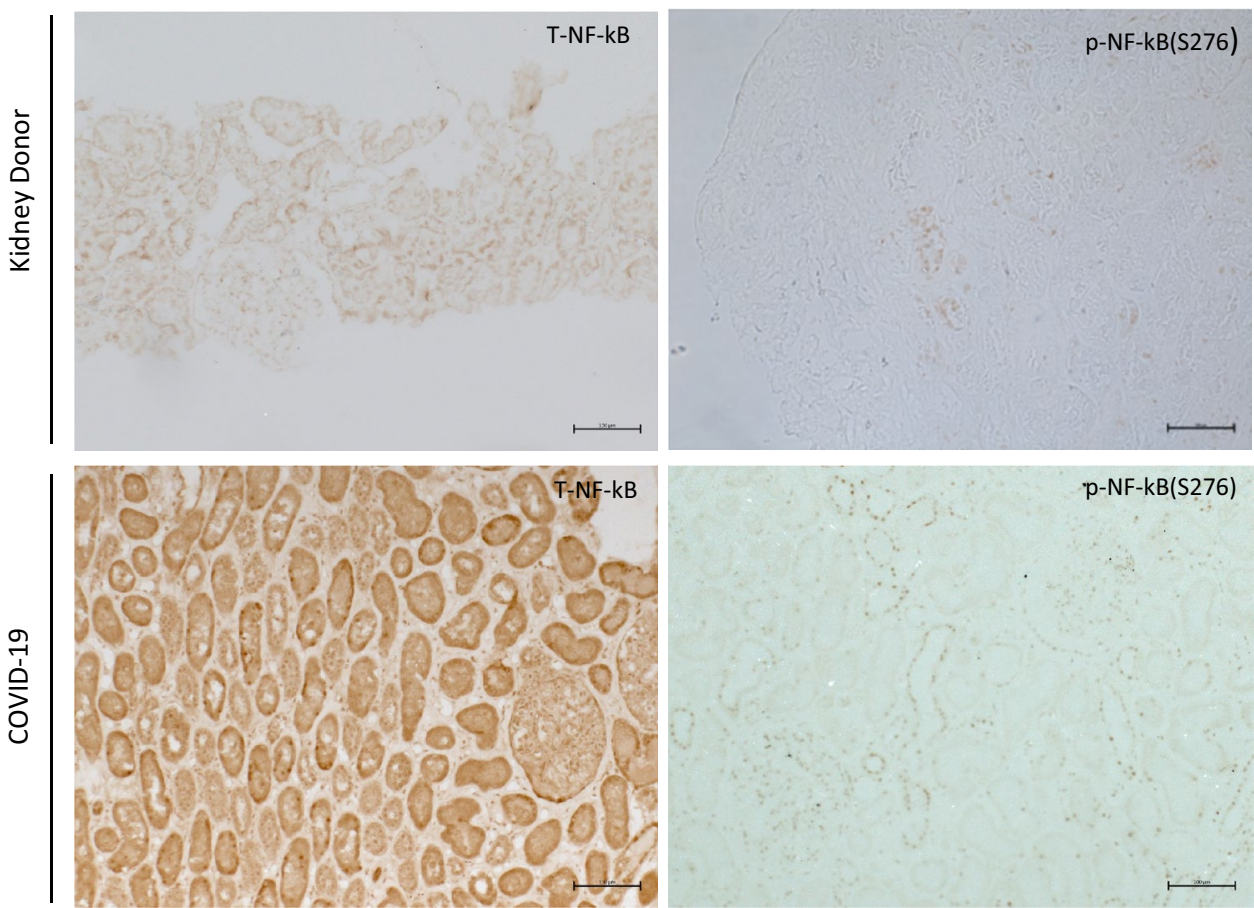

p-NF-kB(S276) a transient or low rate of infection may occur in the kidney cells of some patients. Therefore, SARS-CoV-2 viral proteins or its accessory proteins may also induce activation of STAT3 and NF-kB in the kidney cells after infection.

Interestingly, we found that serine phosphorylation of STAT3 increased in the interstitium of 9/11 AKI cases, while tyrosine phosphorylation of STAT3 and NF-kB phosphorylation occurred in only a few patients. This suggests that the mechanisms mediating the activation of these signaling pathways are likely different. It is known that tyrosine phosphorylation of STAT3 is induced mostly by cytokine-mediated activation of the JAK pathway, while serine phosphorylation of STAT3 is mediated by the MAPK pathway [34]. In addition to the obligatory requirement for tyrosine phosphorylation to activate STAT3, a single serine phosphorylation at residue 727 in the transcriptional activation domain is required for full transcriptional activation of STAT3 [35, 36]. In a previous study, mice with mutations of serine 727 had 50\% reduced STAT3 activity as compared to the wildtype mice and developed less severe diabetic nephropathy [37]. Therefore, we believe that induction of serine 727 phosphorylation is critical for the full activation of STAT3 to induce kidney cell injury in COVID-19 patients. Additionally, STAT3 phosphorylation is also increased in lung tissue, suggesting that this might be a common pathological pathway activated by SARS-CoV-2 in different organs.

Our data suggest that activation of both STAT3 and NF-kB pathways may contribute to kidney injury in some patients with COVID-19. It has been shown that the STAT3 pathway has close crosstalk with the NF-kB pathway to activate downstream gene activation via a positive feedback loop [38, 39]. Therefore, activation of both pathways may lead to more severe kidney cell injury in these patients.

There are several limitations of the current study. We could not observe a direct relationship between the intensity of staining and the severity of AKI in this autopsy series. This is likely due to the small sample size and semi-quantitative nature of the immunostaining. Also, we do not know if the increased STAT3 phosphorylation was due to cytokine storm or to both cytokine storm and local viral infection. Another limitation was that we did not have full cytokine profiles in these patients.

In summary, in one of the largest kidney autopsy series from patients with COVID-19, we identified both glomerular and tubular injury in these patients. We did not find obvious viral infection in the kidney cells by immunofluorescence, electron microscopy, and in-situ hybridization. We demonstrated a distinct activation pattern of STAT3 phosphorylation at tyrosine and serine sites as well as NF-kB phosphorylation in the kidney of these patients. Our study suggests that inhibition of JAK-STAT3 and NF-kB pathways could be potential therapies for AKI in COVID-19 patients. Recently, the FDA authorized the use of baricitinib (Olumiant), an oral Janus kinase (JAK) inhibitor, in combination with intravenous remdesivir (Veklury) to treat hospitalized patients with severe COVID-19 [40]. It would be interesting to see whether this combined therapy will also reduce the incidence and severity of AKI in these patients with severe COVID-19 complications. 
Supplementary Information The online version contains supplementary material available at https://doi.org/10.1007/s40620-021-01173-0.

Author contributions FS, SMJ, LL, and RR analyzed kidney pathology; XL and FZ performed staining for STAT3 and NF-kB, JH and NMC performed analysis of clinical data, and LC and JCH designed the study and wrote the manuscript.

Funding LC is supported in part by the NIH (K23DK124645). $\mathrm{XL}$ is supported by National Nature Science Foundation of China (81970622).

\section{Declarations}

Conflict of interest The authors declare that they have no competing financial interests. The results presented in this paper have not been published previously in whole or part, except in abstract format.

Ethical approval All procedures performed in studies involving human participants were in accordance with the ethical standards of the institutional and/or national research committee and with the 1964 Helsinki declaration and its later amendments or comparable ethical standards. For this retrospective study, formal consent was not required.

\section{References}

1. Chan L, Chaudhary K, Saha A, Chauhan K, Vaid A, Zhao S et al (2020) AKI in Hospitalized Patients with COVID-19. J Am Soc Nephrol. https://doi.org/10.1681/ASN.2020050615

2. Su H, Yang M, Wan C, Yi LX, Tang F, Zhu HY et al (2020) Renal histopathological analysis of 26 postmortem findings of patients with COVID-19 in China. Kidney Int. https://doi.org/10.1016/j. kint.2020.04.003

3. Farkash EA, Wilson AM, Jentzen JM (2020) Ultrastructural Evidence for Direct Renal Infection with SARS-CoV-2. J Am Soc Nephrol. https://doi.org/10.1681/ASN.2020040432

4. Kudose S, Batal I, Santoriello D, Xu K, Barasch J, Peleg Y et al (2020) Kidney biopsy findings in patients with COVID-19. J Am Soc Nephrol 31(9):1959-1968

5. Wu H, Larsen CP, Hernandez-Arroyo CF, Mohamed MMB, Caza T, Sharshir M et al (2020) AKI and collapsing glomerulopathy associated with COVID-19 and APOL 1 high-risk genotype. J Am Soc Nephrol 31(8):1688-1695

6. Nadim MK, Forni LG, Mehta RL, Connor MJ, Liu KD, Ostermann $\mathrm{M}$ et al (2020) COVID-19-associated acute kidney injury: consensus report of the 25th Acute Disease Quality Initiative (ADQI) Workgroup. Nat Rev Nephrol. https://doi.org/10.1038/ s41581-020-00356-5

7. Akilesh S, Nast CC, Yamashita M, Henriksen K, Charu V, Troxell ML et al (2021) Multicenter clinicopathologic correlation of kidney biopsies performed in COVID-19 patients presenting with acute kidney injury or proteinuria. Am J Kidney Dis 77(1):82-93

8. Puelles VG LM, Lindenmeyer MT, Sperhake JP, Wong MN, Allweiss L, Chilla S, Heinemann A, Wanner N, Liu S, Braun F, Lu S, Pfefferle S, Schröder AS, Edler C, Gross O, Glatzel M, Wichmann D, Wiech T, Kluge S, Pueschel K, Aepfelbacher M, Huber TB (2020) Multiorgan and Renal Tropism of SARS-CoV-2. N Engl J Med 383:590-592. https://doi.org/10.1056/NEJMc2015435

9. Puelles VGLM, Lindenmeyer MT, Sperhake JP, Wong MN, Allweiss L, Chilla S, Heinemann A, Wanner N, Liu S, Braun F, Lu S, Pfefferle S, Schröder AS, Edler C, Gross O, Glatzel M, Wichmann D, Wiech T, Kluge S, Pueschel K, Aepfelbacher M, Huber TB
(2020) Multiorgan and renal tropism of SARS-CoV-2. N Engl J Med 383(6):590-592

10. Farkash EA, Wilson AM, Jentzen JM (2020) Ultrastructural Evidence for Direct Renal Infection with SARS-CoV-2. J Am Soc Nephrol. https://doi.org/10.1681/ASN.2020040432

11. Su H, Yang M, Wan C, Yi LX, Tang F, Zhu HY et al (2020) Renal histopathological analysis of 26 postmortem findings of patients with COVID-19 in China. Kidney Int 98(1):219-227

12. François G, Sophie I, Aurélie S, Hassan T, Thomas V, Clarisse $\mathrm{G}$ et al (2020) Tubuloreticular inclusions in COVID-19-related collapsing glomerulopathy. Kidney Int. https://doi.org/10.1016/j. kint.2020.04.022

13. Batlle D, Soler MJ, Sparks MA, Hiremath S, South AM, Welling PA et al (2020) Acute kidney injury in COVID-19: emerging evidence of a distinct pathophysiology. J Am Soc Nephrol 31(7):1380-1383

14. Verdecchia P, Cavallini C, Spanevello A, Angeli F (2020) The pivotal link between ACE2 deficiency and SARS-CoV-2 infection. Eur J Intern Med 76:14-20

15. Brosius FC, Tuttle KR, Kretzler M (2016) JAK inhibition in the treatment of diabetic kidney disease. Diabetologia 59(8):1624-1627

16. Song N, Thaiss F, Guo L (2019) NFkappaB and kidney injury. Front Immunol 10:815

17. Hariharan A, Hakeem AR, Radhakrishnan S, Reddy MS, Rela M (2020) The role and therapeutic potential of NFkappa-B pathway in severe COVID-19 Patients. Inflammopharmacology. https://doi. org/10.1007/s10787-020-00773-9

18. Golmai P, Larsen CP, DeVita MV, Wahl SJ, Weins A, Rennke HG et al (2020) Histopathologic and ultrastructural findings in postmortem kidney biopsy material in 12 patients with AKI and COVID-19. J Am Soc Nephrol 31(9):1944-1947

19. Santoriello D, Khairallah P, Bomback AS, Xu K, Kudose S, Batal I et al (2020) Postmortem kidney pathology findings in patients with COVID-19. J Am Soc Nephrol 31(9):2158-2167

20. Sharma P, Uppal NN, Wanchoo R, Shah HH, Yang Y, Parikh R et al (2020) COVID-19-associated kidney injury: a case series of kidney biopsy findings. J Am Soc Nephrol 31(9):1948-1958

21. Wu Z, McGoogan JM (2020) Characteristics of and important lessons from the coronavirus disease 2019 (COVID-19) outbreak in china: summary of a report of 72314 cases from the Chinese center for disease control and prevention. JAMA 323(13):1239-1242

22. Shetty AA, Tawhari I, Safar-Boueri L, Seif N, Alahmadi A, Gargiulo R et al (2021) COVID-19-associated glomerular disease. J Am Soc Nephrol 32(1):33-40

23. Donnahoo KK, Meng X, Ayala A, Cain MP, Harken AH, Meldrum DR (1999) Early kidney TNF-alpha expression mediates neutrophil infiltration and injury after renal ischemia-reperfusion. Am J Physiol 277(3):R922-R929

24. Meldrum KK, Meldrum DR, Meng X, Ao L, Harken AH (2002) TNF-alpha-dependent bilateral renal injury is induced by unilateral renal ischemia-reperfusion. Am J Physiol Heart Circ Physiol 282(2):H540-H546

25. Markowitz GS, Nasr SH, Stokes MB, D’Agati VD (2010) Treatment with IFN-alpha, -beta, or -gamma is associated with collapsing focal segmental glomerulosclerosis (vol 5, pg 607, 2010). Clin J Am Soc Nephrol 5(7):1353-1453

26. Markowitz GS, Nasr SH, Stokes MB, D'Agati VD (2010) Treatment with IFN-alpha, -beta, or -gamma Is Associated with Collapsing Focal Segmental Glomerulosclerosis (vol 5, pg 607, 2010). Clin Jo American Soc Nephrol 5:1353-1353

27. Dube S, Matam T, Yen J, Mang HE, Dagher PC, Hato T et al (2017) Endothelial STAT3 modulates protective mechanisms in a mouse ischemia-reperfusion model of acute kidney injury. $\mathrm{J}$ Immunol Res 2017:4609502 
28. Zhou Y, Xu W, Zhu H (2019) CXCL8(3-72) K11R/G31P protects against sepsis-induced acute kidney injury via NF-kappaB and JAK2/STAT3 pathway. Biol Res 52(1):29

29. Dube S, Matam T, Yen J, Mang HE, Dagher PC, Hato T et al (2017) Endothelial STAT3 Modulates Protective Mechanisms in a Mouse Ischemia-Reperfusion Model of Acute Kidney Injury. J Immunol Res 2017:4609502. https://doi.org/10.1155/2017/46095 02

30. Schmid H, Boucherot A, Yasuda Y, Henger A, Brunner B, Eichinger $\mathrm{F}$ et al (2006) Modular activation of nuclear factor-kappaB transcriptional programs in human diabetic nephropathy. Diabetes 55(11):2993-3003

31. Wiggins JE, Patel SR, Shedden KA, Goyal M, Wharram BL, Martini $S$ et al (2010) NFkappaB promotes inflammation, coagulation, and fibrosis in the aging glomerulus. J Am Soc Nephrol 21(4):587-597

32. Brosius FC, He JC (2015) JAK inhibition and progressive kidney disease. Curr Opin Nephrol Hypertens 24(1):88-95

33. Bienaime F, Muorah M, Yammine L, Burtin M, Nguyen C, Baron W et al (2016) Stat 3 controls tubulointerstitial communication during CKD. J Am Soc Nephrol 27(12):3690-3705

34. Levy DE, Lee CK (2002) What does Stat3 do? J Clin Invest 109(9):1143-1148

35. Wen Z, Darnell JE Jr (1997) Mapping of Stat3 serine phosphorylation to a single residue (727) and evidence that serine phosphorylation has no influence on DNA binding of Stat1 and Stat3. Nucleic Acids Res 25(11):2062-2067

36. Decker T, Kovarik P (2000) Serine phosphorylation of STATs. Oncogene 19(21):2628-2637

37. Lu TC, Wang ZH, Feng X, Chuang PY, Fang W, Shen Y et al (2009) Knockdown of Stat3 activity in vivo prevents diabetic glomerulopathy. Kidney Int 76(1):63-71

38. Yoshida Y, Kumar A, Koyama Y, Peng H, Arman A, Boch JA et al (2004) Interleukin 1 activates STAT3/nuclear factor-kappaB cross-talk via a unique TRAF6- and p65-dependent mechanism. J Biol Chem 279(3):1768-1776

39. Yu Z, Kone BC (2004) The STAT3 DNA-binding domain mediates interaction with NF-kappaB p65 and inducible nitric oxide synthase transrepression in mesangial cells. J Am Soc Nephrol 15(3):585-591

40. Kalil AC, Patterson TF, Mehta AK, Tomashek KM, Wolfe CR, Ghazaryan V et al (2020) Baricitinib plus Remdesivir for Hospitalized Adults with Covid-19. N Engl J Med. https://doi.org/10. 1056/NEJMoa2031994

Publisher's Note Springer Nature remains neutral with regard to jurisdictional claims in published maps and institutional affiliations. 